\title{
Adaptation to form distortion of a familiar shape
}

\author{
ANN O'LEARY \\ Rutgers University, New Brunswick, New Jersey \\ and \\ MARGO MCMAHON \\ Ursinus College, Collegeville, Pennsylvania
}

\begin{abstract}
Adaptation to the shape distortion produced by an arrangement of cylindrical lenses was demonstrated. Adaptation was produced by exposing subjects to a series of photographs of faces, which appeared horizontally stretched when seen through the lens arrangement. A 16-min adaptation procedure caused a change when the width of a test face was gradually varied and the subject indicated that it looked normal. An alteration in the perceived shape of a test face and an ellipse was also demonstrated when subjects gave estimates of the height and width of these test objects. These studies provide evidence for the operation of memory representations as the veridical cue in process assimilation.
\end{abstract}

When two cues for a perceptual parameter, such as distance, depth, or location with respect to the observer, give conflicting information concerning the value of that parameter, one or both cues, over time, will undergo an alteration in registered value so as to come into closer agreement. This "process assimilation"' has been demonstrated for the nonveridical cue, the veridical one, and both cues (Wallach \& Frey, 1972; Wallach, Frey, \& Bode, 1972; Wallach \& Huntington, 1973). For example, perceived distance that is based on oculomotor cues can be altered by putting the cues into conflict with head movement parallax, with the expansion pattern accompanying approach, and with familiar size. In the latter case, altering the size of a familiar object that is always one particular size, such as a playing card or a dollar bill, makes it a nonveridical distance cue-the perceptual system "assumes" that object to be its usual size and assigns perceived distance accordingly. That familiar size can function as a distance cue is especially interesting, because it demonstrates clearly that memory representations can play an important role in fundamental perceptual processes.

This paper reports an adaptation in form perception that is based on memory representations of the shape of human faces. Monocular exposure to a series of photographs of faces that were made optically wider by an arrangement of cylindrical lenses resulted in a compensatory change in perceived shape, based on a conflict between the shapes of faces that were perceived during adaptation

This work was supported by Grant 11089 from the National Institute of Mental Health to Swarthmore College (Hans Wallach, principal investigator) and by a National Science Foundation graduate fellowship and a Rutgers University Research Council grant to the first author. The authors would like to thank Krista Horlitz, Gillian Rhodes, and Hans Wallach for their helpful discussions and comments. Correspondence should be addressed to Ann O'Leary, Department of Psychology, Tillett Hall, Kilmer Campus, Rutgers University, New Brunswick, NJ 08903. and the remembered shapes of faces encountered in the past. This adaptation was measured with two tests: In the normality-judgment test, the subject's task was to adjust the width of a face so that it looked normal. The shapeestimation test measured a change in the perceived shape of a face, or of an ellipse, by obtaining estimates of width and height. Adaptation was always produced by having the subjects view a series of photographs through the lens arrangement, but the conditions of testing varied from one experiment to another.

\section{EXPERIMENT 1}

In this experiment, the normality-judgment test was used.

\section{Method}

Subjects. Twenty college students were paid to serve as subjects.

Equipment. Distortion was produced with a lens arrangement analogous to a Galilean telescope constructed with cylindrical lenses. This system minified the vertical dimension at .69 power, resulting in an apparent expansion of the horizontal dimension by a factor of 1.45. During adaptation, subjects viewed through the scope a series of photographs of male faces taken from a college yearbook. These faces were selected to be vertically and frontally arranged; that is, none of the faces were tilted to the side, and all had untextured white backgrounds. Each photograph measured approximately $13 \times 8 \mathrm{~cm}$ and was mounted on the front of a light box and transilluminated $1 \mathrm{~m}$ from the subject. Seventeen different photographs were shown during adaptation, another one was used in a practice test, and yet another was used in the normality-judgment test. There was also a drawing a circle, $7.5 \mathrm{~cm}$ in diameter, to be used in the test and a drawing of a square to be used as a practice figure.

In the normality-judgment test, the width of the test face or of the circle could be varied by placing the light box to which they were attached on a turntable so that the rotation axis passed through the center of the photograph or coincided with the vertical diameter of the circle. The width of the test face or of the circle could be changed by rotating the turntable. They were seen through an aperture, either a tall oblong or an ellipse, that obscured the edges of the photographs or of the drawing. As a result, the turned picture 
was perceived not at a slant but in frontoparallel orientation, and changing the rotation angle made it appear wider or narrower. It was viewed through the scope and, when it was to appear with normal width, had to be given some slant to compensate for the effect of the scope.

A scale on the edge of the turntable was calibrated in cosine steps of the rotation angle, because the cosine of the picture's slant angle is linearly related to the projective width of the picture. The cosine scale was multiplied by 100 so that the steps ranged from 1 to 100 . At the 100 setting, the picture was in the subject's frontal plane and was fully stretched by the scope. A stationary pointer was used to read the cosine of the rotation angle. At a width setting of 69, the slant of the picture compensated for the effect of the scope, and the picture was given with normal width.

Procedure. First the subject performed a normality-judgment test by using the square practice figure. It was shown on frontal orientation, where it looked like a horizontal oblong. Then, the wide limit of perceived squareness was found. In this and all other tests involving normality judgments, the experimenter rotated the turntable continuously at a rate of 1 cosine step/sec, each step causing a width change of $1.45 \%$. The subject was instructed to watch the oblong get narrower and to tell the experimenter when it looked square. Next, a practice face was mounted on the light box and turned by the experimenter until the subject reported that it looked normal. Then, the procedure was repeated with the test face or the circle in place, and the wide limit of the normality range was obtained where the face or the circle first looked normal.

After the wide limit of the range of normality or circularity had been identified, the narrow limit of each was obtained. The initial width setting in the narrowing of the figures was .40 . This test order was chosen because it seemed likely that the subjects' seeing the narrow versions of the test figures that were presented to obtain the narrow limits would greatly diminish the adaptation effect by, in effect, producing a condition that was opposite to the one that had led to adaptation. For 10 subjects, the test was done first with the face and then with the circle; for the other 10 , the order was reversed.

In the adaptation period, each of the 17 photographs was shown for $\mathbf{4 0} \mathrm{sec}$ in frontal orientation, where the scope increased its width by a factor of 1.45 . Seven of the photographs were shown a second time at random points in the square. The subject reported whenever a previously presented picture was shown-no easy task, because the shape distortion was quite strong. This was done to ensure that the subject would attend fully to the faces. For a given subject, the order of testing after adaptation was the same as before.

\section{Results}

The mean limits of the width ranges at which the face appeared normal or the circle circular are listed in Table 1. Data obtained for the face and the circle, when they were presented first and second, are listed separately. The combined scores are also given. That our procedure yielded accurate judgments can be seen when the preadaptation ranges are compared with the width value at which the effect of the scope was compensated for, namely, at the 69 width setting. The mean midpoints between the means of the wide and the narrow limits were at the width settings of 65.9 and 65.1 for the face and the circle, respectively.

An adaptation effect was obtained only for the face, but there it was a large one. A much wider face appeared to be normal after, as opposed to before, adaptation. When the face test was performed before the circle test, before dissipation of the adaptation effect could occur, the wide limit was 10.9 cosine steps wider after the adaptation period than it was before; this effect was highly signifi-
Table 1

Mean Wide and Narrow Limits of Normality Range of Test Face and Circle Before and After Adaptation in Experiment 1

\begin{tabular}{|c|c|c|c|c|c|}
\hline & \multicolumn{5}{|c|}{ Presentation Order } \\
\hline & $n$ & Before & After & Difference & $t$ \\
\hline Limits: & & & & & \\
\hline $\begin{array}{l}\text { Wide } \\
\text { Face first } \\
\text { Face second } \\
\text { Combined }\end{array}$ & $\begin{array}{l}10 \\
10 \\
20\end{array}$ & $\begin{array}{l}74.70 \\
73.70 \\
74.20\end{array}$ & $\begin{array}{l}85.59 \\
78.70 \\
82.14\end{array}$ & $\begin{array}{r}10.89 \\
5.00 \\
7.94\end{array}$ & $\begin{array}{l}4.92 \ddagger \\
3.74 \dagger \\
5.55 \ddagger\end{array}$ \\
\hline $\begin{array}{l}\text { Narrow } \\
\text { Face first } \\
\text { Face second } \\
\text { Combined }\end{array}$ & $\begin{array}{l}10 \\
10 \\
20\end{array}$ & $\begin{array}{l}56.63 \\
58.67 \\
57.65\end{array}$ & $\begin{array}{l}55.82 \\
62.07 \\
58.95\end{array}$ & $\begin{array}{r}-.81 \\
3.40 \\
1.30\end{array}$ & $\begin{array}{l}\text { n.s. } \\
2.17^{*} \\
\text { n.s. }\end{array}$ \\
\hline $\begin{array}{l}\text { Wide } \\
\text { Circle first } \\
\text { Circle second } \\
\text { Combined }\end{array}$ & $\begin{array}{l}10 \\
10 \\
20\end{array}$ & $\begin{array}{l}71.49 \\
71.23 \\
71.36\end{array}$ & $\begin{array}{l}70.06 \\
70.79 \\
70.42\end{array}$ & $\begin{array}{r}-1.43 \\
-.57 \\
-.94\end{array}$ & $\begin{array}{l}\text { n.s. } \\
\text { n.s. } \\
\text { n.s. }\end{array}$ \\
\hline $\begin{array}{l}\text { Narrow } \\
\text { Circle first } \\
\text { Circle second } \\
\text { Combined } \\
\end{array}$ & $\begin{array}{l}10 \\
10 \\
20 \\
\end{array}$ & $\begin{array}{l}60.63 \\
57.02 \\
58.82 \\
\end{array}$ & $\begin{array}{l}60.73 \\
56.80 \\
58.77 \\
\end{array}$ & $\begin{array}{r}.10 \\
-.22 \\
-.05 \\
\end{array}$ & $\begin{array}{l}\text { n.s. } \\
\text { n.s. } \\
\text { n.s. }\end{array}$ \\
\hline
\end{tabular}

cant $[t(9)=4.92, p<.001]$. The narrow limit did not reliably demonstrate an adaptation effect; presumably, unlearning occurred when the very narrow face was presented in the test. When testing began with the circle, dissipation presumably occurred while the circle test was being performed and resulted in a marked attenuation of the face adaptation effect. ${ }^{1}$ The difference between pretest and posttest for the wide limit was reduced to 5.0 cosine steps. This was significantly smaller than the effect measured immediately after the adaptation period $[t(18)=4.8$, $p<.001]$.

There was no effect of exposure to wide faces on the limits of perceived circularity. The effect for the test face was 12.3 cosine steps larger than the effect measured with the circle test; this difference was significant $[t(18)=$ 9.36, $p<.001]$.

Thus, the results of Experiment 1 indicate that, following exposure to a series of much wider than normal faces, a wider test face is judged as normal than is the case before such an exposure. There seem to be two possible explanations for this result:

1. Based on the conflict between the much wider faces observed during the adaptation period and the memory of faces previously perceived under ordinary conditions, a compensatory change in form perception occurs such that faces are perceived as narrower than they are. As a consequence, after adaptation, an objectively wider face is accepted as normal.

2. The much wider faces seen in the adaptation period leave memories that, added to the memories of normal faces, shift the norm for face shapes so that a wider test face is accepted as normal.

Our next experiment was a direct test of whether our adaptation condition would result in a change in form perception. To determine whether the test face was perceived 
as narrower after adaptation than it was before, we obtained estimates of the width and the height of the test face before and after the adaptation period.

\section{EXPERIMENT 2}

\section{Method}

Subjects. Forty-eight college students were paid to serve as subjects.

Equipment. In addition to the photographs, scope, and the apparatus used in Experiment 1, a device was designed to obtain estimates of width and height of the test face. A white line was drawn on an oblong black board parallel to its longer edges. Retaining slots fastened to these edges held two black slats that could be moved toward or away from each other, revealing a longer or shorter section of the white line. By adjusting the distance between the inner edges of the slats, the subject could make the white line equivalent in length to the dimension of the face to be estimated. The subjects held the board on their laps, keeping the white line in horizontal orientation. To make an estimate, they looked alternately at the face through the scope and directly down at the board, and they adjusted the slats until the white line appeared to be as long as the width or the height of the face. The line was in horizontal orientation for both width and height estimates.

Procedure. Thirty-two subjects served in the experimental condition and 16 subjects served as a control group. For the former, the adaptation period was the same as in Experiment 1. The control group viewed the photographs directly, that is, through a hole, instead of through the scope. Otherwise, the adaptation period was the same for the two groups. In addition to the shape-estimation tests, normality-judgment tests were also performed.

At the outset, each subject practiced the two tests on a square that looked rectangular when seen through the scope. First, width and height estimates were obtained for the square at the 95 width setting. Then, by using the procedure of Experiment 1, the wide limit for perceived squareness was found. Next, a practice face was presented, and the wide limit of perceived normal shape was obtained. Then, the preadaptation tests with the actual test face were performed. First, the shape-estimation test was given, with the face at the $\mathbf{9 5}$ width setting. It was followed by the normality-judgment test, in which the wide limit of the perceived normal shape was obtained. Then, the two tests were repeated in the same order. Finally, the normal width setting obtained in the second normalityadjustment test was used to obtain width and height estimates for the face shape that appeared to the subject to be normal. In all shapeestimation tests performed in this study, the order in which width and height estimates were obtained was counterbalanced.

Following the adaptation period, one shape-estimation test and one normality-judgment test were given in rapid succession. To control for the effects of test order, half of the experimental subjects underwent the shape-estimation test before the normality-judgment test and half were tested in the opposite sequence. After these tests, the normal width setting obtained in the just-performed normalityjudgment test was used to obtain postadaptation width and height estimates for the face that appeared to the subject to be normal after the adaptation period.

Testing for the 16 control subjects was the same, except that only one order of testing was used after the adaptation period: the shapeestimation test always came first.

\section{Results}

The results of the shape-estimation tests for the experimental and the control groups are listed in Table 2, with the results for the two preadaptation tests averaged. They show a clear effect. To the experimental subjects, the test face looked narrower after adaptation than it did
Table 2

Means and Standard Deviations of Shape Estimates (in $\mathrm{cm}$ ) in Experiment 2

\begin{tabular}{|c|c|c|c|c|c|c|c|}
\hline & \multirow[b]{2}{*}{ Setting } & \multirow[b]{2}{*}{$n$} & \multicolumn{2}{|c|}{ Width } & \multicolumn{2}{|c|}{ Height } & \multirow{2}{*}{$\begin{array}{l}\text { Width: } \\
\text { Height }\end{array}$} \\
\hline & & & $M$ & $S D$ & $M$ & $S D$ & \\
\hline \multicolumn{8}{|c|}{ Experimental Group } \\
\hline $\begin{array}{l}\text { Preexposure } \\
\text { Postexposure } \\
\text { Difference }\end{array}$ & $\begin{array}{l}95 \\
95\end{array}$ & $\begin{array}{l}32 \\
32 \\
32\end{array}$ & $\begin{array}{l}8.30 \\
7.76 \\
0.54\end{array}$ & $\begin{array}{l}3.6 \\
3.5\end{array}$ & $\begin{array}{l}9.27 \\
9.20 \\
0.07\end{array}$ & $\begin{array}{l}4.0 \\
4.0\end{array}$ & $\begin{array}{l}.90 \\
.84 \\
.06^{*}\end{array}$ \\
\hline \multicolumn{8}{|c|}{ Control Group } \\
\hline $\begin{array}{l}\text { Preexposure } \\
\text { Postexposure } \\
\text { Difference }\end{array}$ & $\begin{array}{l}95 \\
95\end{array}$ & $\begin{array}{l}16 \\
16 \\
16\end{array}$ & $\begin{array}{l}7.06 \\
6.86 \\
0.20\end{array}$ & $\begin{array}{l}3.5 \\
3.4\end{array}$ & $\begin{array}{l}7.82 \\
7.65 \\
0.17\end{array}$ & $\begin{array}{l}4.1 \\
3.9\end{array}$ & $\begin{array}{l}.90 \\
.90 \\
0\end{array}$ \\
\hline
\end{tabular}

${ }^{*} p<.001$.

before. The width-to-height ratio had changed from .90 before adaptation to .84 after adaptation. This difference was significant $[t(31)=3.93, p<.001]$. The testing order after adaptation had little effect. The width-to-height ratio was .066 when the shape-estimation test followed adaptation directly and .052 when the normality-judgment test was given first, with this difference not significant $[t(30)=.37]$. No change in the width-to-height ratio was found for the control group.

The results of the normality-judgment tests appear in Table 3 in the rows labeled Limit. Only the results of the second pretest are presented. ${ }^{2}$ As in the result of this test in Experiment 1, the wide limit of normality was significantly wider after the adaptation period than it was before, but the change was much smaller than it was in Experiment $1-2.39$ cosine steps compared with 7.95 cosine steps in Experiment $1 .{ }^{3}$ This difference may, in part, be due to the fact that in Experiment 1 normality-judgment tests were the only measure of adaptation, whereas in Experiment 2 they followed shape-estimation tests. It seems possible that the analytic approach to form called for by the width and height estimates of the shape-estimation tests made the subjects more conservative when assessing "normality." The difference in the preadaptation means of the wide limits of the normality ranges measured in Experiments 1 and 2 , that is, 74.20 versus 78.58 , may have been due to a

Table 3

Mean Normality Judgments for the Wide Limits and Mean Shape Estimates at These Limits (in cm) with Standard Deviations in Experiment 2

\begin{tabular}{|c|c|c|c|c|c|c|}
\hline \multirow[b]{2}{*}{$n$} & & \multicolumn{2}{|c|}{ Preexposure } & \multicolumn{2}{|c|}{ Postexposure } & \multirow[b]{2}{*}{ Difference } \\
\hline & & $M$ & $S D$ & $M$ & $S D$ & \\
\hline \multicolumn{7}{|c|}{ Experimental Group } \\
\hline 32 & $\begin{array}{l}\text { Limit } \\
\text { Width } \\
\text { Height }\end{array}$ & $\begin{array}{r}78.58 \\
6.86 \\
9.27\end{array}$ & $\begin{array}{l}7.4 \\
3.1 \\
4.0\end{array}$ & $\begin{array}{r}80.97 \\
6.81 \\
9.14\end{array}$ & $\begin{array}{l}7.9 \\
3.1 \\
4.0\end{array}$ & $2.39 *$ \\
\hline & Width:Height & .75 & & .75 & & 0 \\
\hline \multicolumn{7}{|c|}{ Control Group } \\
\hline 16 & $\begin{array}{l}\text { Limit } \\
\text { Width } \\
\text { Height } \\
\text { Width:Height }\end{array}$ & $\begin{array}{r}78.20 \\
6.10 \\
7.84 \\
.77\end{array}$ & $\begin{array}{l}5.0 \\
3.5 \\
4.1\end{array}$ & $\begin{array}{r}77.90 \\
6.09 \\
7.89 \\
.77\end{array}$ & $\begin{array}{l}3.8 \\
3.1 \\
4.1\end{array}$ & -0.30 \\
\hline
\end{tabular}


Table 4

Means and Standard Deviations of Shape Estimates (in $\mathrm{cm}$ ) in Experiment 3

\begin{tabular}{|c|c|c|c|c|c|c|}
\hline & \multirow[b]{2}{*}{$n$} & \multicolumn{2}{|c|}{ Width } & \multicolumn{2}{|c|}{ Height } & \multirow{2}{*}{$\begin{array}{l}\text { Width } \\
\text { Heigh }\end{array}$} \\
\hline & & $M$ & $S D$ & $M$ & $S D$ & \\
\hline \multicolumn{7}{|c|}{ Binocular Group } \\
\hline Preexposure & 16 & 7.48 & 2.2 & 8.71 & 2.1 & .86 \\
\hline Postexposure & 16 & 7.89 & 2.8 & 9.65 & 3.3 & .82 \\
\hline Difference & 16 & -0.41 & & -0.94 & & $.04 *$ \\
\hline
\end{tabular}

${ }^{*} p<.01$.

norm shift that occurred during the presentation of the wide face for shape estimates; this may have been another factor militating against apparent change in the normality estimate. In the control group of Experiment 2, there was, as expected, no change in the normality-judgment test.

The other rows of Table 3 list the mean width and height estimates obtained for the test face at the wide limits of perceived normality. As would be expected, the preexposure shape estimates differ from those shown in Table 2, much as the limits of perceived normality differ, namely $16 \%$ and $17 \%$, respectively. Corroborating the results for the shape-estimation test made at the wide setting are those for the "perceived normal" face. Although the actual retinal shapes of the faces at the normality settings made before and after adaptation differ, the shape estimates in width-to-height ratios do not, indicating that the wider postadaptation normal face is seen as having the same shape as the preadaptation one.

\section{EXPERIMENT 3}

The adaptation effect observed in Experiment 2 presumably involves some recalibration of the correspondence between perceived space and the mapping of the scene on the retina and brain, perhaps serving naturalistically/ evolutionarily to compensate for changes in the retina caused by growth or injury. It seemed possible that the adaptation effect might occur only in connection with monocular exposure to the lens. With the use of two lenses, a replication of Experiment 2 was performed under conditions of binocular viewing. The procedure was very similar to that of Experiment 2, except that subjects made the size estimates with binocular vision.

\section{Method}

Sixteen undergraduates served as subjects. Width and height estimates were practiced: first, by using a circle, which, seen through the scopes, looked like an ellipse at the 95 width setting, and then by using a practice face. To obtain the preadaptation estimates, a face different from the one employed in Experiment 2 was viewed through the scopes at the 95 width setting. The adaptation procedure was the same as in Experiment 2 and was immediately followed by the postadaptation shape estimates.

\section{Results}

The mean width and height estimates before and after adaptation are listed in Table 4 . These means differ from the corresponding ones listed in Table 2 because a different test face was used, but approximately the same adaptation effect was measured. The difference of .04 between the pre- and postadaptation width-to-height ratios was comparable to the .06 ratio difference that was obtained in Experiment 2 and was significant $[t(15)=3.45, p<.01]$. The results of this experiment confirmed those of the corresponding test in Experiment 2.

\section{EXPERIMENT 4}

In Experiment 1, where adaptation was measured with the normality-judgment test, when the test face was replaced by a circle, no adaptation effect was obtained, indicating that the effect did not generalize to nonface stimuli. However, we wished to test for generalization once more with the more stringent shape-estimation test. Once again, we used a circle stimulus, which, at the wide setting, was an ellipse. The experiment resembled Experiment 2 and used monocular exposures. We did not deem it necessary to include a control group in this experiment.

\section{Method}

Sixteen undergraduates served as subjects. They first made height and width estimates for the practice square that looked rectangular when seen through the scope. Then, the wide limit of perceived squareness was found. Next, the preadaptation tests were performed. Height and width estimates were obtained for the drawing of a circle, $7.5 \mathrm{~cm}$ in diameter, presented at the 95 width setting, at which it was optically an ellipse. Following this shape-estimation test, the wide limit of circularity was obtained. The two tests were then repeated in the same order. Following the standard adaptation procedure, each of the tests was administered once, again with the shapeestimation test first and the normality-judgment test second.

\section{Results}

The results for Experiment 4 are listed in Table 5. After adaptation, the width-to-height ratio decreased from 1.49 to 1.38 ; this difference was significant $[t(15)=3.42$, $p<.01]$. The .11 decrease in the width-to-height ratio

Table 5

Means and Standard Deviations of Shape Estimates (in cm) for an Ellipse and of Wide Limits of Normality Range of a Circular Shape in Experiment 4

\begin{tabular}{|c|c|c|c|c|c|c|c|c|c|}
\hline & \multirow{3}{*}{$\begin{array}{l}\text { Width } \\
\text { Setting }\end{array}$} & \multirow[b]{3}{*}{$n$} & \multicolumn{4}{|c|}{ Shape Estimates } & \multirow{3}{*}{$\begin{array}{l}\text { Width: } \\
\text { Height }\end{array}$} & \multirow{2}{*}{\multicolumn{2}{|c|}{$\begin{array}{l}\text { Wide Limits } \\
\text { of Normality }\end{array}$}} \\
\hline & & & \multicolumn{2}{|c|}{ Width } & \multicolumn{2}{|c|}{ Height } & & & \\
\hline & & & $M$ & $S D$ & $M$ & $S D$ & & $M$ & $S D$ \\
\hline Preex & 95 & 16 & 9.35 & 3.7 & 6.26 & 2.8 & 1.49 & 72.2 & 5.6 \\
\hline Postexposure & 95 & 16 & 9.08 & 3.0 & 6.58 & 3.6 & 1.38 & 73.8 & 7.13 \\
\hline Difference & & & 0.27 & & -0.32 & & $.11^{*}$ & 1.6 & \\
\hline
\end{tabular}

${ }^{*} p<.01$. 
agrees well with the .06 decrease obtained for the test face in Experiment 2. The former represents a $6.7 \%$ and the latter a $7.4 \%$ change. The mean wide limit of circularity obtained in the normality-judgment test changed little. The difference of 1.6 cosine steps between the second preadaptation test and the postadaptation test was not significant $[t(15)=1.54, p<.1]$. This negative result is in agreement with the failure of an identical test in Experiment 1 to show any adaptation effect, although the null hypothesis cannot be accepted with confidence, given the $t$ value and the sample size.

\section{SUMMARY AND DISCUSSION}

Lengthy exposure to a number of optically distorted faces resulted in an adaptation such that perception compensated partly for their conspicuous horizontal stretch. A test face is perceived as narrower after the exposure than it was before, and this effect could be measured by two tests. In the normality-judgment test, the test face was gradually made narrower until the subject judged its shape to be normal. After exposure to the stretched faces, that is, after the adaptation period, a substantially wider face was accepted as normal than was accepted before (Experiment 1). In the shape-estimation test, estimates of the width and of the height of the optically stretched test face were obtained before and after the adaptation period. After the adaptation period, the width-to-height ratio was smaller than it was before, indicating that the test face was then perceived as narrower (Experiment 2). That the shapeestimation test measured a change in the perceptual process is confirmed by the finding that it need not measure the shape of a face. When width and height estimates for an ellipse were made before and after exposure to horizontally stretched faces, a change in the width-to-height ratio was obtained in an amount quite similar to the one obtained for a stretched face (Experiment 4).

This change in the perceptual process that causes a horizontally stretched face, as well as an ellipse, to be seen as narrower can be regarded as compensation for the excessive width of the faces seen in the adaptation period. It diminishes the discrepancy between the faces seen in the adaptation period and the memories of normal faces, and thus resembles other cases of perceptual adaptation that result from cue conflicts (chap. 10 in Wallach, 1976). ${ }^{4}$

This change in the perceptual process also accounts for a change measured by the normality-judgment test. When, after adaptation, a test face is perceived as narrower, an objectively wider face will look normal. Whereas change in the perceptual process thus contributes to the adaptation measured by the normality-judgment test, it does not fully explain the result that was obtained with this test. The effect measured with the normality-judgment test was much larger than the perceptual change measured with the shape-estimation test. The change in the wide limit in the normality range of 10.89 cosine steps amounts to a change of $14.6 \%$, whereas the change of .06 in the width-to-height ratio measured in Experiment 2 amounts to a change of $6.7 \%$. As stated above, it is possible that normality judgments are different after adaptation, because the much wider faces seen in the adaptation period leave memories that, added to the memories of normal faces, shift temporarily the norm for face shapes so that a wider test face is accepted as normal. Inasmuch as the norm shift operates independently of the change in shape perception, the effects of the two may add, and this may account for the large shift in the normality range.

The assumption that the adaptation effect measured by the normality-judgment test is not entirely due to a norm shift, and is, in part, a change in shape perception, seems to be contradicted by the finding that the normalityjudgment test showed, twice, in Experiments 1 and 4, no adaptation effect when the test figure was a circle. Inasmuch as a change in shape perception could have been measured with the shape-estimation test when the test figure was not a face but an ellipse, why was it not measured with the normality-judgment test when the test figure was a circle, when this test is assumed to measure, in part, a change in shape perception? A circle does not differ from an ellipse only by its width and height being equal but also by its constant curvature. If the latter is its dominant characteristic, it would not be altered by a change in shape perception that alters the proportion of width and height. A different test shape, one with a vertical-symmetry axis only, may have given a different result. This is an instance in which adaptation in form perception differs from adaptation of simple parameters.

\section{REFERENCES}

Wallach, H. (1976). Hans Wallach on perception. New York: Times Books.

Wallach, H., \& Frey, K. J. (1972). On counteradaptation. Perception \& Psychophysics, 11, 161-165.

Wallach, H., FreY, K. J., \& BODE, K. A. (1972). The nature of adaptation in distance perception based on oculomotor cues. Perception \& Psychophysics, 11, 110-116.

Wallach, H., Huntington, D. (1973). Counteradaptation after exposure to displaced visual direction. Perception \& Psychophysics, 13, 519-524.

\section{NOTES}

1. It would be interesting in future research to compare the rate of unlearning during exposure to narrow faces with that of spontaneous dissipation, for example, that which might occur in darkness.

2. The subjects' pretest trials were not averaged, because it was discovered that the limit of perceived normal shape increased from the first pretest to the second. Thus, on the first repetition of a normality judgment, the subjects tended to shift to a wider setting, perhaps due to a tendency for subjects to be overly conservative on the first trial. Scores for the control subjects indicate that no such artifactual shift could account for the experimental findings, since no further increase was observed between judgments made at the second pretest and the posttest.

3. The 7.95 cosine step is the average of the mean obtained when the face test was taken first and second.

4. Presumably, when this process operates under naturalistic conditions involving retinal growth or injury, the compensation process would occur in the dimension(s) of actual deformation of the retina.

(Manuscript received December 22, 1989; revision accepted for publication October 1, 1990.) 